\title{
Hasse Principle for the Chow Groups of Zero-cycles on Quadric Fibrations
}

\author{
Kazuki SATO \\ Tohoku University \\ (Communicated by N. Suwa)
}

\begin{abstract}
We give a sufficient condition for the injectivity of the global-to-local map of the relative Chow group of zero-cycles on a quadric fibration of dimension $\leq 3$ defined over a number field.
\end{abstract}

\section{Introduction}

Let $k$ be a number field and $\Omega$ the set of its places. For any variety $X$ over $k, \mathrm{CH}_{0}(X)$ denotes the Chow group of zero-cycles on $X$ modulo rational equivalence. Then we have the global-to-local map

$$
\mathrm{CH}_{0}(X) \longrightarrow \prod_{v \in \Omega} \mathrm{CH}_{0}\left(X \otimes_{k} k_{v}\right),
$$

where for each place $v \in \Omega, k_{v}$ denotes the completion of $k$ at $v$. If there exists a proper morphism $X \rightarrow C$ from $X$ to another variety $C$, we also have the relative version of the global-to-local map

$$
\Phi: \mathrm{CH}_{0}(X / C) \longrightarrow \prod_{v \in \Omega} \mathrm{CH}_{0}\left(X \otimes_{k} k_{v} / C \otimes_{k} k_{v}\right),
$$

where $\mathrm{CH}_{0}(X / C)$ denotes the kernel of the push-forward map $\mathrm{CH}_{0}(X) \rightarrow \mathrm{CH}_{0}(C)$. In this paper, we study the injectivity of $\Phi$ for quadric fibrations over curves.

First, let us recall some known results for a surface $X$. In the case where $X$ is a conic bundle surface over the projective line $\mathbf{P}_{k}^{1}$, Salberger proved that there exists the following exact sequence of finite abelian groups

$$
0 \longrightarrow \amalg^{1}(k, T) \longrightarrow A_{0}(X) \stackrel{\Phi}{\longrightarrow} \bigoplus_{v \in \Omega} A_{0}\left(X \otimes_{k} k_{v}\right) \longrightarrow \operatorname{Hom}\left(H^{1}(k, \hat{T}), \mathbf{Q} / \mathbf{Z}\right),
$$

Received April 3, 2013; revised March 13, 2014 
where $A_{0}(X)$ is the kernel of the degree homomorphism deg: $\mathrm{CH}_{0}(X) \rightarrow \mathbf{Z}$ (see [7] for the details).

In the case where $X$ is a quadric fibration of dimension $\geq 4$, few results are known. Parimala and Suresh proved that if $X \rightarrow C$ is a quadratic fibration over a smooth projective curve $C$ whose generic fiber is defined by a Pfister neighbor of rank $\geq 5$, then the global-tolocal map restricted to real places

$$
\Phi_{\text {real }}: \mathrm{CH}_{0}(X / C) \longrightarrow \bigoplus_{v: \text { real place }} \mathrm{CH}_{0}\left(X \otimes_{k} k_{v} / C \otimes_{k} k_{v}\right)
$$

is injective [6]. By using this injectivity, they deduced a finiteness result of the torsion subgroup of the Chow group $\mathrm{CH}_{0}(X)$ of zero-cycles on $X$.

When $X \rightarrow C$ is a quadric fibration of $\operatorname{dim} \leq 3$, not only that the map $\Phi_{\text {real }}$ is not injective in general, but also the map $\Phi$ is not injective [8]. However, the map $\Phi$ can be injective. If the generic fiber of $X \rightarrow C$ is defined by a quadratic form over a base field $k$, the map $\Phi$ is injective (Theorem 3.1). The above condition does not imply the injectivity of $\Phi_{\text {real }}$, and we give an example of this (Proposition 3.3). Note that we do not assume that quadric fibrations are admissible (for the definition of admissibility, see [6]).

NOTATION AND CONVENTIONS. In section $2, k$ denotes a field of characteristic different from 2. In section $3, k$ denotes a number field (i.e. a finite extension field of $\mathbf{Q}$ ). For a variety $X,|X|$ denotes the set of closed points on $X$. We denote by $\mathrm{CH}_{i}(X)$ the Chow group of cycles of dimension $i$ on $X$ modulo rational equivalence [3]. For a geometrically integral variety $X$ over $k, k(X)$ denotes the function field of $X$. For any extension $L / k$ of fields, $L(X)$ denotes the function field of $X \otimes_{k} L$. If $x$ is a point in $X, k(x)$ denotes the residue field at $x$.

\section{Definition of the map $\delta$}

In this section, for a quadric fibration $X \rightarrow C$ (see definition 2.2), we define a quotient group $k(C)^{*} / k^{*} N_{q}(k(C))$ and a homomorphism

$$
\delta: \mathrm{CH}_{0}(X / C) \longrightarrow k(C)^{*} / k^{*} N_{q}(k(C)) .
$$

We will see that the homomorphism $\delta$ is injective, and our main theorem is based on this property.

First, we recall definitions and some properties about a quadratic space.

By a quadratic space over $k$, we mean a nonsingular quadratic form over $k$. We denote by $W(k)$ the Witt group of quadratic spaces over $k$ and by $I k$ the fundamental ideal of $W(k)$ consisting of classes of even rank quadratic spaces. We represent quadratic spaces over $k$ by diagonal matrices $\left\langle a_{1}, \ldots, a_{n}\right\rangle\left(a_{i} \in k^{*}\right)$ with respect to the choice of an orthogonal basis. By an $n$-fold Pfister form over $k$, we mean a quadratic space of the type $\left\langle 1, a_{1}\right\rangle \otimes\left\langle 1, a_{2}\right\rangle \otimes \cdots \otimes$ $\left\langle 1, a_{n}\right\rangle$. The set of nonzero values of a Pfister form is a subgroup of the multiplicative group $k^{*}$ of $k$ [5, Theorem 1.8, p. 319]. By a Pfister neighbor of an $n$-fold Pfister form $q$, we mean a quadratic space of rank at least $2^{n-1}+1$ which is a subform of $q$ [4, Example 4.1]. 
For any quadratic space $q$ over $k$, let $N_{q}(k)$ be the subgroup of $k^{*}$ generated by norms from finite extensions $E$ of $k$ such that $q$ is isotropic over $E$. If $q$ is isotropic, then clearly $N_{q}(k)=k^{*}$. For any $a \in k^{*}, q$ is isotropic if and only if $\langle a\rangle \otimes q$ is isotropic. Therefore $N_{q}(k)=N_{\langle a\rangle \otimes q}(k)$. By Knebusch's norm principle, $N_{q}(k)$ is generated by elements of the form $x y$, with $x, y \in k^{*}$ which are values of $q$ over $k$ [2, Lemme 2.2]. In particular, if a quadratic form $q$ is of the form $q=\langle 1, a\rangle \otimes\langle 1, b\rangle$, then $N_{q}(k)$ is equal to the group $\operatorname{Nrd}_{D / k}\left(D^{*}\right)$ of reduced norms of the quaternion algebra $D=(-a,-b)_{k}$. Suppose that $q^{\prime}$ is a Pfister neighbor of a Pfister form $q$. Then, for any extension $E / k, q^{\prime}$ is isotropic over $E$ if and only if $q$ is isotropic over $E$ [4, Example 4.1]. So we have $N_{q}(k)=N_{q^{\prime}}(k)$.

The following lemma is elementary and well-known, but the proof does not seem to be written explicitly in the literature.

Lemma 2.1. Let $q$ be a Pfister form over $k$. Then

$$
N_{q}(k)=\left\{x \in k^{*} \mid q \otimes\langle 1,-x\rangle \text { is isotropic }\right\} .
$$

In particular, $x$ belongs to $N_{q}(k)$ if and only if $q \otimes\langle 1,-x\rangle=0$ in $W(k)$.

Proof. Since $q$ is a Pfister form, $N_{q}(k)$ is the set of non-zero values of $q$. If $q$ is isotropic, the assertion is clear. We suppose that $q$ is anisotropic. If $q \otimes\langle 1,-x\rangle$ is isotropic, then there exist two vectors $v_{1}, v_{2}$ in the underlying vector space of $q$, not both zero, such that $q\left(v_{1}\right)-x q\left(v_{2}\right)=0$. Therefore

$$
x=q\left(v_{1}\right) / q\left(v_{2}\right) \in N_{q}(k) .
$$

The other implication follows from the fact that a Pfister form represents 1 .

The last assertion results from the basic fact on Pfister forms [5, Theorem 1.7, p. 319].

DEFINITION 2.2. Let $C$ be a smooth projective geometrically integral curve over $k$. A quadric fibration $(X, \pi)$ over $C$ is a geometrically integral variety $X$ over $k$, together with a proper flat $k$-morphism $\pi: X \rightarrow C$ such that each point $P$ of $C$ has an affine neighborhood Spec $A(P)$, with $X \times_{C}$ Spec $A(P)$ isomorphic to a quadric in $\mathbf{P}_{A(P)}^{n}$ and such that the generic fiber of $\pi$ is a smooth quadric.

Given a quadratic space $q$ over the function field $k(C)$ of $C$ of rank $n+1 \geq 3$, we can easily construct a quadric fibration $\pi: X \rightarrow C$, whose generic fiber is given by the quadratic space $q$. Indeed, for the choice of an orthogonal basis the quadratic space $q$ corresponds to a homogeneous polynomial $q\left(t_{0}, t_{1}, \ldots, t_{n}\right)$ of degree two with coefficients in $k(C)$. We define the smooth quadric hypersurface $Q$ by

$$
Q=\left\{q\left(t_{0}, t_{1}, \ldots, t_{n}\right)=0\right\} \subset \operatorname{Proj}\left(k(C)\left[t_{0}, t_{1}, \ldots, t_{n}\right]\right)=\mathbf{P}_{k(C)}^{n} .
$$

Let $X$ be the scheme-theoretic image of the morphism $Q \hookrightarrow \mathbf{P}_{k(C)}^{n} \rightarrow \mathbf{P}_{k}^{n} \times_{\operatorname{Spec} k} C$. By composition with the second projection $\mathbf{P}_{k}^{n} \times \operatorname{spec} k C \rightarrow C$, we get a quadric fibration $X \rightarrow C$, 
whose generic fiber is $Q$. Conversely, for any quadric fibration $\pi: X \rightarrow C$, the generic fiber of $\pi$ is a smooth quadric hypersurface over $k(C)$. Its defining equation gives a quadric space $q$ over $k(C)$, which is well-defined up to multiplication by a non-zero element of $k(C)$. However, as mentioned above, the group $N_{q}(k(C))$ is independent of the choice of $q$.

Let $\pi: X \rightarrow C$ be a quadric fibration with the generic fiber given by a quadratic space $q$, and $\mathrm{CH}_{0}(X / C)$ denote the kernel of the map

$$
\pi_{*}: \mathrm{CH}_{0}(X) \longrightarrow \mathrm{CH}_{0}(C) \text {. }
$$

We have the following commutative diagram with exact rows (see [2, Proposition 1.1])



where $X_{\eta}$ is the generic fiber of $\pi: X \rightarrow C$ and $\operatorname{deg}_{X_{P} / k(P)}: \mathrm{CH}_{0}\left(X_{P}\right) \rightarrow \mathbf{Z}$ is the degree map. Since the map $\bigoplus_{x \in\left|X_{\eta}\right|} k(x)^{*} \rightarrow k(C)^{*}$ is induced by norms, the image is precisely $N_{q}(k(C))$. By the snake lemma and the fact that $A_{0}\left(X_{P}\right)=0$ [9], we have an exact sequence

$$
0 \longrightarrow \mathrm{CH}_{0}(X / C) \stackrel{\delta}{\longrightarrow} k(C)^{*} / k^{*} N_{q}(k(C)) \longrightarrow \bigoplus_{P \in C^{(1)}} \mathbf{Z} / \operatorname{deg}_{X_{P} / k(P)}\left(\mathrm{CH}_{0}\left(X_{P}\right)\right) .
$$

REMARK 2.3. We denote by $k(C)_{\mathrm{dn}}^{*}(q)$ the subgroup of $k(C)^{*}$ consisting of functions which, at each closed point $P \in C$, can be written as a product of a unit at $P$ and an element of $N_{q}(k(C))$. Colliot-Thélène and Skorobogatov [2, Théorème 1.4] proved that the above homomorphism $\delta$ induces an isomorphism

$$
\delta: \mathrm{CH}_{0}(X / C) \stackrel{\sim}{\longrightarrow} k(C)_{\mathrm{dn}}^{*}(q) / k^{*} N_{q}(k(C))
$$

for an admissible quadric fibration $\pi: X \rightarrow C$. However, we do not have to assume the admissibility for our main results. Indeed, in this paper, we consider only the injectivity of the global-to-local map for the relative Chow group $\mathrm{CH}_{0}(X / C)$. On the other hand, the map $\delta: \mathrm{CH}_{0}(X / C) \rightarrow k(C)^{*} / k^{*} N_{q}(k(C))$ is injective whether $\pi: X \rightarrow C$ is admissible or not. Therefore we can reduce the injectivity of the global-to-local map for the relative chow group $\mathrm{CH}_{0}(X / C)$ to the injectivity of the global-to-local map for the group $k(C)^{*} / k^{*} N_{q}(k(C))$.

\section{Injectivity of the global-to-local map}

Let $k$ be a number field and $\Omega$ be the set of places of $k$. For any $v \in \Omega, k_{v}$ denotes the completion of $k$ at $v$. 
In [6, Theorem 5.4], Parimala and Suresh proved that if $X \rightarrow C$ is an admissible quadric fibration whose generic fiber is given by a Pfister neighbor over $k(C)$ of rank $\geq 5$, then the map

$$
\Phi_{\text {real }}: \mathrm{CH}_{0}(X / C) \longrightarrow \bigoplus_{v: \text { real places }} \mathrm{CH}_{0}\left(X \otimes_{k} k_{v} / C \otimes_{k} k_{v}\right)
$$

is injective, where $v$ runs over all real places of $k$. In the case where the rank of the quadratic form defining the generic fiber is less than 5 , we give a sufficient condition for the injectivity of the global-to-local map $\Phi$.

THEOREM 3.1. Let $\pi: X \rightarrow C$ be a quadric fibration over a number field $k$. Assume that $\operatorname{dim} X=2$ or 3 , and the generic fiber of $\pi$ is isomorphic to a quadric defined over $k$ (i.e. there exists a quadric $Q \subset \mathbf{P}_{k}^{N}$ such that the generic fiber is isomorphic to $Q \otimes_{k} k(C)$ over $k(C))$. Then, the natural map

$$
\Phi: \mathrm{CH}_{0}(X / C) \longrightarrow \bigoplus_{v \in \Omega} \mathrm{CH}_{0}\left(X \otimes_{k} k_{v} / C \otimes_{k} k_{v}\right)
$$

is injective.

PROOF. Let $q$ be a quadratic form defining the generic fiber of the quadric fibration $\pi: X \rightarrow C$. In order to prove the theorem, it is sufficient to show that the natural map

$$
k(C)^{*} / k^{*} N_{q}(k(C)) \longrightarrow \prod_{v \in \Omega} k_{v}(C)^{*} / k_{v}^{*} N_{q}\left(k_{v}(C)\right)
$$

is injective (see Remark 2.3).

First, when $\operatorname{dim} X=3$, that is, $\operatorname{rank} q=4$, we show that the global-to-local map $\Phi$ is injective and for almost all $v$ the $v$-component $\mathrm{CH}_{0}\left(X \otimes_{k} k_{v} / C \otimes_{k} k_{v}\right)$ is 0 .

We may assume that $q=\langle 1, a, b, a b d\rangle, a, b, d \in k^{*}$. Put $L:=k(\sqrt{d})$. Note that $q$ is isometric to $\langle 1, a\rangle \otimes\langle 1, b\rangle$ over $L(C)$. Let $f \in k(C)^{*}$ be an element which belongs to $k_{v}^{*} N_{q}\left(k_{v}(C)\right)$ for all places $v$ of $k$. For any real place $w$ of $L$, denote by $w^{\prime}$ the restriction of $w$ to $k$. Since $f \in k_{w^{\prime}}^{*} N_{q}\left(k_{w^{\prime}}(C)\right)$, there exists $\mu_{w^{\prime}} \in k_{w^{\prime}}^{*}$ such that $\mu_{w^{\prime}} f \in N_{q}\left(k_{w^{\prime}}(C)\right)$. We can choose $\mu \in k^{*}$ such that the sign of $\mu$ is the same as that of $\mu_{w^{\prime}}$ for each real place $w$ of $L$. Thus we have $\mu f \in N_{q}\left(k_{w^{\prime}}(C)\right) \subset N_{q}\left(L_{w}(C)\right)$. Therefore $q \otimes\langle 1,-\mu f\rangle$ is hyperbolic over $L_{w}(C)$ for each real place $w$ of $L$. For a complex place $w$ of $L$, it is clear that $q \otimes\langle 1,-\mu f\rangle$ is hyperbolic over $L_{w}(C)$. Further, for a finite place $w$ of $L$, we have $f \in k_{v}^{*} N_{q}\left(k_{v}(C)\right)$, where $v$ is the place of $k$ below $w$. Since $k_{v}^{*} \subset N_{q}\left(k_{v}(C)\right.$ ) (this follows from the fact that $k_{v}^{*}=N_{q}\left(k_{v}\right)$ for any quadratic form $q$ of rank at least 3 over a $p$-adic field $k_{v}$ ),

$$
\mu f \in k_{v}^{*} N_{q}\left(k_{v}(C)\right)=N_{q}\left(k_{v}(C)\right) \subset N_{q}\left(L_{w}(C)\right) .
$$

Therefore $q \otimes\langle 1,-\mu f\rangle$ is hyperbolic over $L_{w}(C)$ for all places $w$ of $L$. By [1, Theorem 4], 
the natural map

$$
I^{3} L(C) \longrightarrow \prod_{w} I^{3} L_{w}(C)
$$

is injective, where $w$ runs over all places of $L$. Hence $q \otimes\langle 1,-\mu f\rangle$ is hyperbolic over $L(C)$. By [2, Proposition 2.3], we have

$$
\mu f \in N_{q}(L(C)) \cap k(C)^{*}=N_{q}(k(C)) .
$$

This proves the required injectivity.

The image of the global-to-local map $\Phi$ lies in the direct sum $\bigoplus_{v} \mathrm{CH}_{0}\left(X \otimes_{k} k_{v} / C \otimes_{k} k_{v}\right)$. Indeed, a quadratic form of rank 4 defined over a number field $k$ is isotropic over $k_{v}$ for all but finitely many places $v$ of $k$. Therefore for all but finitely many $v, \mathrm{CH}_{0}\left(X \otimes_{k} k_{v} / C \otimes_{k} k_{v}\right)=0$.

Finally, we consider the case $\operatorname{dim} X=2$, that is, $\operatorname{rank} q=3$.

We can assume $q=\langle 1, a, b\rangle, a, b \in k^{*}$. Since $q$ is a Pfister neighbor of the Pfister form $q^{\prime}=\langle 1, a\rangle \otimes\langle 1, b\rangle$, we have $N_{q}(k(C))=N_{q^{\prime}}(k(C))$ [4, Example 4.1]. Therefore by the above case the map

$$
k(C)^{*} / k^{*} N_{q}(k(C)) \longrightarrow \prod_{v \in \Omega} k_{v}(C)^{*} / k_{v}^{*} N_{q}\left(k_{v}(C)\right)
$$

is injective. This completes the proof.

REMARK 3.2. Without the assumption that the generic fiber is defined over $k$, the natural map

$$
\Phi: \mathrm{CH}_{0}(X / C) \longrightarrow \prod_{v \in \Omega} \mathrm{CH}_{0}\left(X \otimes_{k} k_{v} / C \otimes_{k} k_{v}\right)
$$

is not injective [8].

Finally, we consider the restricted global-to-local map $\Phi_{\text {real }}$. Parimala and Suresh's result [6, Theorem 5.4], which is concerned with quadratic forms of rank at least 5, does not hold for forms of smaller rank. We give the following example, which is a variant of [6, Proposition 6.1].

PROPOSITION 3.3. Let $C$ be the elliptic curve over $\mathbf{Q}$ defined by

$$
y^{2}=-x(x+2)(x+3) .
$$

Assume that the generic fiber of a quadric fibration $\pi: X \rightarrow C$ is isomorphic to the quadric defined by the quadratic form $q=\langle 1,-2,3,-6\rangle$. Then the natural map

$$
\Phi_{\text {real }}: \mathrm{CH}_{0}(X / C) \longrightarrow \mathrm{CH}_{0}\left(X \otimes_{\mathbf{Q}} \mathbf{R} / C \otimes_{\mathbf{Q}} \mathbf{R}\right)
$$

is not injective. 
Proof. Since $q$ is isotropic over $\mathbf{R}, N_{q}(\mathbf{R}(C))=\mathbf{R}(C)^{*}$. So we have $\mathrm{CH}_{0}\left(X \otimes_{\mathbf{Q}}\right.$ $\left.\mathbf{R} / C \otimes_{\mathbf{Q}} \mathbf{R}\right)=0$.

Since we have $\operatorname{div}_{C}(x)=2 D$ for some divisor $D$ on $C, x \in \mathbf{Q}(C)^{*} / \mathbf{Q}^{*} N_{q}(\mathbf{Q}(C))$ is contained in $\operatorname{Im} \delta$. On the other hand, $q$ is isometric to $\langle 1,1,3,3\rangle$ over $\mathbf{Q}_{3}$. Thus $x \notin$ $\mathbf{Q}_{3}^{*} N_{q}\left(\mathbf{Q}_{3}(C)\right)$ by [6, Proposition 6.1]. Therefore we have $\mathrm{CH}_{0}(X / C) \neq 0$.

REMARK 3.4. In the above case the map

$$
\Phi: \mathrm{CH}_{0}(X / C) \longrightarrow \bigoplus_{v \in \Omega} \mathrm{CH}_{0}\left(X \otimes_{\mathbf{Q}} \mathbf{Q}_{v} / C \otimes_{\mathbf{Q}} \mathbf{Q}_{v}\right)
$$

is injective by Theorem 3.1. The summands of the right hand side vanish except for $\mathrm{CH}_{0}\left(X \otimes_{\mathbf{Q}} \mathbf{Q}_{2} / C \otimes_{\mathbf{Q}} \mathbf{Q}_{2}\right)$ and $\mathrm{CH}_{0}\left(X \otimes_{\mathbf{Q}} \mathbf{Q}_{3} / C \otimes_{\mathbf{Q}} \mathbf{Q}_{3}\right)$, since the quadratic form $\langle 1,-2,3,-6\rangle$ is isotropic over $\mathbf{R}$ and over $\mathbf{Q}_{p}$ for all primes $p$ except 2 and 3.

ACKNowledgment. I would like to thank Professor Masaki Hanamura for helpful comments and warm encouragement. I also would like to express my gratitude to the referee for several comments and suggestions. This work was supported by JSPS KAKENHI Grant Number $25 \cdot 1135$.

\section{References}

[ 1 ] J. Kr. Arason, R. Elman, and B. Jacob, Fields of cohomological 2-dimension three, Math. Ann. 274 (1986), no. 4, 649-657.

[ 2 ] J.-L. Colliot-Thélène and A. N. Skorobogatov, Groupe de Chow des zéro-cycles sur les fibrés en quadriques, $K$-Theory 7 (1993), no. 5, 477-500.

[ 3 ] W. Fulton, Intersection theory, 2nd ed., Ergebnisse der Mathematik und ihrer Grenzgebiete. 3. Folge. A Series of Modern Surveys in Mathematics [Results in Mathematics and Related Areas. 3rd Series. A Series of Modern Surveys in Mathematics], vol. 2, Springer-Verlag, Berlin, 1998.

[ 4 ] M. Knebusch, Generic splitting of quadratic forms. I, Proc. London Math. Soc. (3) 33 (1976), no. 1, 65-93.

[ 5 ] T. Y. LAM, Introduction to quadratic forms over fields, Graduate Studies in Mathematics, vol. 67, American Mathematical Society, Providence, RI, 2005.

[6] R. Parimala and V. Suresh, Zero-cycles on quadric fibrations: finiteness theorems and the cycle map, Invent. Math. 122 (1995), no. 1, 83-117.

[ 7 ] P. SAlberger, Zero-cycles on rational surfaces over number fields, Invent. Math. 91 (1988), no. 3, 505-524.

[ 8 ] V. SURESH, Zero cycles on conic fibrations and a conjecture of Bloch, $K$-Theory 10 (1996), no. 6, 597-610,

[ 9 ] R. G. SwAN, Zero cycles on quadric hypersurfaces, Proc. Amer. Math. Soc. 107 (1989), no. 1, $43-46$.

Present Address:

Mathematical Institute,

TOHOKU UNIVERSITY,

SENDAI, MiYAGI 980-8578, JAPAN.

e-mail:sb0m17@math.tohoku.ac.jp 